\title{
The Effect of Body Weight and Alcohol Consumption on Hyperuricemia and Their Population Attributable Fractions: A National Health Survey in China
}

\author{
Huijing He ${ }^{a}$ Li Pan ${ }^{a}$ Xiaolan Ren ${ }^{b}$ Dingming Wang ${ }^{c}$ Jianwei Du ${ }^{d} \quad$ Ze Cuie \\ Jingbo Zhao ${ }^{f}$ Hailing Wang ${ }^{g}$ Xianghua Wang ${ }^{\text {h }}$ Feng Liu ${ }^{i}{\text { Lize } \mathrm{Pa}^{\mathrm{j}}}$ \\ Xia Peng ${ }^{k}$ Ye Wang ${ }^{a}$ Chengdong Yu ${ }^{a}$ Guangliang Shan ${ }^{a}$
}

aDepartment of Epidemiology and Statistics, Institute of Basic Medical Sciences, Chinese Academy of Medical

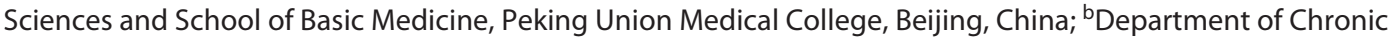
and Noncommunicable Disease Prevention and Control, Gansu Provincial Center for Disease Control and Prevention, Lanzhou, China; 'Department of Chronic and Noncommunicable Disease Prevention and Control, Guizhou Provincial Center for Disease Control and Prevention, Guiyang, China; ${ }^{d}$ Department of Chronic and Noncommunicable Disease Prevention and Control, Hainan Provincial Center for Disease Control and Prevention,

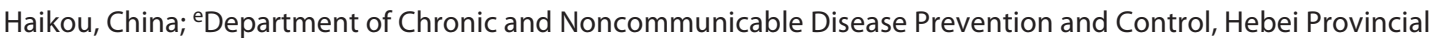
Center for Disease Control and Prevention, Shijiazhuang, China; 'Department of Epidemiology and Statistics, School of Public Health, Harbin Medical University, Harbin, China; ${ }^{9}$ Department of Chronic and Noncommunicable Disease Prevention and Control, Inner Mongolia Autonomous Region Center for Disease Control and Prevention,

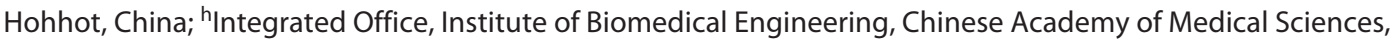
Peking Union Medical College, Tianjin, China; 'Department of Chronic and Noncommunicable Disease Prevention and Control, Shaanxi Provincial Center for Disease Control and Prevention, Xi'an, China; 'Department of Chronic and Noncommunicable Disease Prevention and Control, Xinjiang Uyghur Autonomous Region Center for Disease Control and Prevention, Urumqi, China; ${ }^{k}$ Department of Chronic and Noncommunicable Disease Prevention and Control, Yunnan Provincial Center for Disease Control and Prevention, Kunming, China

\section{Keywords}

Alcohol consumption · Body mass index · Hyperuricemia · Obesity $\cdot$ Risk factors

\begin{abstract}
Introduction: The prevalence of hyperuricemia (HUA) is increasing worldwide; understanding of population attributable fraction of modifiable risk factors (MRFs) is important for disease prevention. Given the sparse evidence on how MRFs influence HUA in mainland China, we aimed to explore the effect of excess body weight and alcohol consumption and their population attributable fractions of HUA based on a na-
\end{abstract}

tional survey in mainland China. Methods: Using data from the China National Health Survey which included 31,746 Han Chinese of 20-80 years of age from 10 provinces, we estimated the prevalence and MRFs (overweight/obesity and alcohol consumption) of HUA. HUA was defined as serum uric acid $>417 \mu \mathrm{mol} / \mathrm{L}$ in men and $>340 \mu \mathrm{mol} / \mathrm{L}$ in women. Restricted cubic-spline models were used to demonstrate the linear and nonlinear associations between exposures and HUA. The adjusted population attributable risk (PAR) was calculated to understand the relative importance of each MRF. Results: The prevalence of HUA was $25.1 \%$ in men and $15.9 \%$ in women. The population fraction of HUA cases that could be avoided by weight loss was $20.6 \%$ (19.3\%-22.0\%)
C 2021 The Author(s).

Published by S. Karger AG, Basel

This is an Open Access article licensed under the Creative Commons Attribution-NonCommercial-4.0 International License (CC BY-NC) (http://www.karger.com/Services/OpenAccessLicense), applicable to the online version of the article only. Usage and distribution for commercial purposes requires written permission.
Correspondence to:

Guangliang Shan, guangliang_shan@163.com 
in men and $18.1 \%(17.1 \%-19.0 \%)$ in women. The PAR of alcohol consumption was $12.8 \%(8.5 \%-17.1 \%)$ in men. Participants from Southwest China (Yunnan) had the highest HUA prevalence $(47.9 \%$ in men and $29.9 \%$ in women) but with lower PAR of MRFs, especially in men (16.7\%). Subjects in North China had lower HUA prevalence but higher PAR of MRFs. Around $44.8 \%$ male HUA cases in Inner Mongolia (26.9\% of HUA prevalence) and $37.7 \%$ cases in Heilongjiang (34.4\% of HUA prevalence) were attributable to overweight/ obesity and alcohol consumption. Conclusion: There are significant sex and geographic difference on PAR of HUA due to MRFs. More tailored prevention strategies are needed to prevent HUA through weight loss and reduction of alcohol consumption.

(C) 2021 The Author(s)

Published by S. Karger AG, Basel

\section{Introduction}

Hyperuricemia (HUA), defined as the presence of elevated serum uric acid (SUA) concentration, is a necessary condition for the development of gout and is associated with metabolic syndrome, insulin resistance, kidney disease, and cardiovascular diseases [1-3]. The prevalence of HUA has been increasing in recent decades [4, 5]. Based on the results of the US National Health and Nutrition Examination Survey (NHANES) 2007-2008, the prevalence of HUA was over $21 \%$ [5].

As a large developing country, China has remarkable regional differences and varied populations [6]. Reported as around $10 \%$ in the general population [7-10], the prevalence of HUA in China varied substantially according to geographic and socioeconomic factors [7]. However, less attention has been paid to the geographic variation of HUA modifiable risk factors (MRFs) and therefore failed to develop tailored interventions within provinces. Risk factors related to dietary and lifestyle changes, such as alcohol consumption and adiposity, were believed to be associated with HUA [9]. Mendelian randomization studies have shown that adiposity and alcohol consumption are causally associated with HUA [11-13]. Lifestyle interventions may bring about normalization of SUA levels [14]. By calculating the population attributable risk (PAR) of overweight/obesity and alcohol consumption (considered as MRFs of HUA in this study), we can obtain the expected proportion of HUA cases that could be prevented if these factors were removed [15]. The relative importance of MRFs in different regions and populations may provide key evidence for public health intervention of HUA and help policy makers to initiate more tailored and area-specific strategies. Therefore, using data from the China National Health Survey (CNHS) [16], we aimed to assess the effect of excess body weight and alcohol consumption on HUA and further explore the population and geographic disparities of their PARs.

\section{Materials and Methods}

\section{Study Design and Subjects}

The CNHS is a nationwide cross-sectional survey conducted from 2012 to 2017. The study protocol and population have been described in detail previously [16]. Briefly, by a stratified, multistage cluster sampling method, adults from 11 provinces of mainland China were recruited. All the participants underwent questionnaire interviews and health examinations. Individuals of 20-80 years of age, who had lived in their current residence for at least 1 year, were eligible to participate. Individuals who had severe mental or physical illness or pregnant or were military personnel with active service were excluded [16]. Our analysis was limited to Han participants who underwent biochemical tests. The final sample included 31,746 adults from 10 provinces (one province was excluded as it contains no Han people) with complete data on the target risk factors (see online suppl. Fig. S1. for more details; for all online suppl. material, see www.karger.com/doi/10.1159/000521163).

The study has been carried out in accordance with the Declaration of Helsinki. Ethical approval was obtained from the Bioethical Committee of Institute of Basic Medical Sciences, Chinese Academy of Medical Sciences (No. 029-2013). All participants provided written informed consent before the survey.

\section{Data Collection and Measurement}

Information on demographics (e.g., age and sex) and socioeconomic factors (urban or rural residence,educational attainment) were collected by a face-to-face questionnaire interview. Physical examination and biomedical measurements were performed at each study site by trained staff based on a standardized field work instruction protocol. Height was measured to the nearest $0.1 \mathrm{~cm}$ using a fixed stadiometer. Weight was measured by a body composition analyzer (BC-420; Tanita, Tokyo, Japan), with the accuracy on a decimal level. Body mass index (BMI) was calculated as weight divided by height squared $\left(\mathrm{kg} / \mathrm{m}^{2}\right)$. Venous blood samples were drawn after an overnight fast. Separated plasma or serum was frozen in aliquots and stored at $-80^{\circ} \mathrm{C}$ until thawed for the first time for the analyses. The SUA level was measured by oxidization with the specific enzyme uricase on a chemistry analyzer (Cobas 8000 C 701; Roche, Indianapolis, IN, USA).

HUA was defined as SUA $>417 \mu \mathrm{mol} / \mathrm{L}$ in men and $>340$ $\mu \mathrm{mol} / \mathrm{L}$ in women based on the NHANES-III laboratory definition [17]. Underweight was defined as $\mathrm{BMI}<18.5 \mathrm{~kg} / \mathrm{m}^{2}$, normal weight was BMI between $18.5 \mathrm{~kg} / \mathrm{m}^{2}$ and $<25 \mathrm{~kg} / \mathrm{m}^{2}$, overweight was BMI $\geq 25 \mathrm{~kg} / \mathrm{m}^{2}$ but $<30 \mathrm{~kg} / \mathrm{m}^{2}$, and obesity was defined as BMI $\geq 30 \mathrm{~kg} /$ $\mathrm{m}^{2}$ [18]. Alcohol drinking was classified into 3 groups: current drinkers, ex-drinkers, and never drinkers. The definition for each group of alcohol consumption has been described by our previous studies $[16,19]$. Based on the World Health Organization's guide for monitoring alcohol consumption and related harm, for males, alcohol consumption level was defined as low (1-40 g/day), medium (41-60 g/day), and high (>60 g/day); for females, the catego- 
ries were 1-20 g/day, 21-40 g/day, and $>40 \mathrm{~g} /$ day, respectively [20].

Other covariates included smoking status and estimated glomerular filtration rate $\left(\mathrm{eGFR}, \mathrm{mL} / \mathrm{min}\right.$ per $\left.1.73 \mathrm{~m}^{2}\right)$. The eGFR was calculated according to the Modification of Diet in Renal Disease equation for Chinese [21]. The formula for eGFR calculation was:

$$
\mathrm{eGFR}=175 \times \mathrm{Scr}^{-1.234} \times \text { age }^{-0.179} \times 0.742(\text { if female })
$$

Scr is serum creatinine in milligram per decilitre. Smoking status was categorized as never smoke and ever smoke (including current smokers and ex-smokers). A current smoker was defined as smoking at least one cigarette per day for at least 6 months at the time of survey. An ex-smoker was defined as stopping smoking for more than 6 months preceding the survey $[16,19]$.

\section{Patient and Public Involvement}

Patients (in this study is participants recruited in a general population) and service providers were involved in various stage of this study. Before the survey, trainings were carried out, and survey bulletins were disseminated to provincial and subdistrict management, facility management, and clinic staff, as to prioritize barriers to survey implementation that needed to be addressed. In the survey, health education was embedded to promote the health literacy among participants, especially the seniors, illiterate people, or people from rural areas. Feedback of the physical examination would be returned to participants in the first place by both online and offline services.

\section{Statistical Analysis}

The final analytical samples were restricted to subjects without missing value of major risk factors (age, sex, and SUA), eGFR $\geq 60$ $\mathrm{mL} / \mathrm{min}$ per $1.73 \mathrm{~m}^{2}$ (to exclude possible kidney disorders [22]), no self-report of gout, or the use of diuretic medication. As SUA varied substantially by sex [23], we analyzed the data by sex separately.

The comparisons of prevalence among groups were adjusted for potential confounders (age and residential areas) using logistic regression models [24]. The survey logistic regression models were used to estimate the effect of excess body weight and alcohol consumption on HUA. In addition, we examined the possible nonlinear relationships between BMI, alcohol daily intake, and HUA using restricted cubic-spline models. Tests for nonlinearity used the likelihood ratio test and compared the model with only the linear term to the model with linear and cubic-spline terms.

Prevalence ratios rather than odds ratios (ORs) were used in the estimation of PAR. Given the relatively high frequency of HUA, the OR may overestimate the value of the association between risk factors and the outcome [25]. For overweight/obesity and alcohol consumption, we calculated their PARs, which were estimates of the percentage of HUA cases in this population that would have been avoided if the risk factors exposure decreased to the lowest risk group (i.e., BMI $<25 \mathrm{~kg} / \mathrm{m}^{2}$ and no alcohol consumption). The following formula was used to calculate PAR:

$$
\mathrm{PAR}=\sum_{i=0}^{k} \mathrm{pd}_{i}\left(\frac{\mathrm{PR}_{\mathrm{i}}-1}{\mathrm{PR}_{\mathrm{i}}}\right)=1-\sum_{i=0}^{k} \frac{\mathrm{pd}_{i}}{\mathrm{PR}_{i}} .
$$

In the above formula, $\mathrm{pd}_{i}$ refers to the proportion of cases in the ${ }_{\mathrm{i}}$ th exposure level, and the prevalence ratio represents the prevalence risk (which is a simulation of relative risk), comparing the ith exposure level with the unexposed group $(i=0) ; k$ refers to the total number of categories of the exposure (in the current study, the exposures were overweight/obesity vs. underweight/normal weight and current alcohol consumption vs. no current alcohol drink) [15]. The adjusted PARs accounted for covariates were obtained by regression models $[26,27]$, which reflect the relative importance of the risk factors. The prevalence of HUA if MRFs were eliminated $=$ overall prevalence of HUA $\times(1-\mathrm{PAR}$ [overweight/ obesity] - PAR [alcohol consumption]).

We performed the statistical analyses using SAS version 9.4 for windows (SAS Institute Inc, Cary, NC, USA). For all estimates, we calculated and presented their $95 \%$ confidence intervals. All $p$ values were derived from two-sided tests.

\section{Results}

\section{Basic Characteristics}

A total of 31,746 adults of 20-80 years of age participated in the study. The mean age was 49.0 years (standard deviation $=13.5$ ) for males and 48.3 years (standard deviation $=13.1$ ) for females. The mean SUA level was 366.9 (interquartile range: $307.7-417.4) \mu \mathrm{mol} / \mathrm{L}$ in men and 277.8 (interquartile range: $231.6-316.3) \mu \mathrm{mol} / \mathrm{L}$ in women. The overall prevalence of HUA was $19.6 \%, 25.1 \%$ in men and $15.9 \%$ in women, respectively. The adjusted prevalence and risk factor distributions are presented in Table 1.

\section{The Prevalence of HUA and Risk Factors}

Females had a lower HUA prevalence than males (Table 1). There was sex difference of the effect of aging on HUA prevalence or risk, i.e., decreased risk in men but increased in women (Fig. 1, 2). Other associated factors of HUA included living in urban areas, having higher education (in men), overweight and obesity, and alcohol drinking (in men) (Fig. 1). In general, comparing with women, men had higher prevalence of overweight/obesity and current alcohol consumption. Areas in North China had higher prevalence of overweight/obesity. The prevalence of current alcohol consumption in men ranged from $47.3 \%$ (Shaanxi province in western China) to $79.8 \%$ (Hebei province in middle China). There were linear and nonlinear relationships between $\mathrm{BMI}$ and the OR of HUA in both sexes (both $p$ values $<0.001$ ). Linear and nonlinear relationships were also found between alcohol daily intake and HUA in men (both $p$ values $<0.001$ ) (Fig. 2).

In either men or women, individuals from North China, i.e., Hebei and Heilongjiang provinces, had higher overweight/obesity adjusted prevalence, with $56.8 \%$ in men from Hebei and $46.0 \%$ in men from Heilongjiang and correspondingly a relatively high adjusted HUA prevalence $(31.9 \%$ and $34.4 \%$, respectively). However, 
Table 1. Prevalence of HUA in adults from the CNHS, 2012-2017 $(n=31,746)$

\begin{tabular}{|c|c|c|c|c|c|c|}
\hline & \multicolumn{3}{|c|}{ Male $(n=12,701)$} & \multicolumn{3}{|c|}{ Female $(n=19,045)$} \\
\hline & $\begin{array}{l}\text { cases of } \\
\text { HUA }\end{array}$ & $\begin{array}{l}\text { crude } \\
\text { prevalence, \% }\end{array}$ & $\begin{array}{l}\text { adjusted prevalence, } \\
\%(95 \% \mathrm{Cl})^{*}\end{array}$ & $\begin{array}{l}\text { cases of } \\
\text { HUA }\end{array}$ & $\begin{array}{l}\text { crude } \\
\text { prevalence, } \%\end{array}$ & $\begin{array}{l}\text { adjusted prevalence, } \\
\%(95 \% \mathrm{Cl})^{*}\end{array}$ \\
\hline Overall & 3,184 & 25.07 & $24.03(23.23-24.85)$ & 3,036 & 15.94 & $15.07(14.52-15.65)$ \\
\hline \multicolumn{7}{|l|}{ Demographic characteristics } \\
\hline$p$ value & & $<0.001$ & $<0.001$ & & $<0.001$ & $<0.001$ \\
\hline \multicolumn{7}{|l|}{ Age-groups, years } \\
\hline $20-$ & 394 & 30.10 & $28.12(25.75-30.61)$ & 247 & 12.06 & $10.95(9.69-12.34)$ \\
\hline $30-$ & 625 & 30.46 & $28.19(26.27-30.19)$ & 316 & 10.05 & $9.30(8.35-10.35)$ \\
\hline $40-$ & 842 & 26.04 & $24.41(22.95-25.93)$ & 583 & 11.35 & $10.58(9.77-11.45)$ \\
\hline $50-$ & 738 & 23.18 & $22.01(20.60-23.49)$ & 933 & 19.65 & $18.63(17.54-19.77)$ \\
\hline \multicolumn{7}{|l|}{ Educational level } \\
\hline High school & 1,558 & 24.00 & $23.07(21.94-24.24)$ & 1,382 & 15.83 & $15.01(14.14-15.93)$ \\
\hline College or above & 1,177 & 30.33 & $24.54(22.94-26.22)$ & 623 & 13.70 & $14.86(13.60-16.22)$ \\
\hline$p$ value & & $<0.001$ & 0.027 & & $<0.001$ & 0.835 \\
\hline \multicolumn{7}{|l|}{ MRFs } \\
\hline \multicolumn{7}{|l|}{ BMI, $\mathrm{kg} / \mathrm{m}^{2}$} \\
\hline$<25$ & 1,261 & 17.44 & $16.34(15.44-17.27)$ & 1,382 & 10.80 & $10.62(10.04-11.23)$ \\
\hline $25-$ & 1,540 & 33.37 & $31.68(30.18-33.23)$ & 1,296 & 25.04 & $23.43(22.16-24.75)$ \\
\hline$\geq 30$ & 349 & 47.81 & $45.10(41.40-48.86)$ & 322 & 36.34 & $34.93(31.74-38.26)$ \\
\hline$p$ value & & $<0.001$ & $<0.001$ & & $<0.001$ & $<0.001$ \\
\hline \multicolumn{7}{|l|}{ Alcohol consumption status } \\
\hline Never drink & 560 & 17.73 & $17.09(15.79-18.47)$ & 2,482 & 15.87 & $14.89(14.25-15.56)$ \\
\hline Quit drink & 304 & 23.96 & $24.14(21.78-26.67)$ & 84 & 22.46 & $19.86(16.18-24.14)$ \\
\hline \multicolumn{7}{|l|}{ Cigarette smoking } \\
\hline Never smoke & 1,039 & 26.26 & $23.41(22.05-24.83)$ & 2,924 & 15.75 & $14.97(14.35-15.60)$ \\
\hline Ever smoke & 2,140 & 24.51 & $23.00(22.00-24.03)$ & 110 & 23.55 & $20.37(17.03-24.17)$ \\
\hline$p$ value & & 0.036 & 0.604 & & $<0.001$ & 0.001 \\
\hline \multicolumn{7}{|l|}{ Locations } \\
\hline Shaanxi & 311 & 13.34 & $12.52(11.23-13.94)$ & 302 & 9.07 & $9.33(8.35-10.40)$ \\
\hline Gansu & 427 & 24.36 & $23.16(21.18-25.28)$ & 403 & 16.36 & $15.26(13.84-16.80)$ \\
\hline Guizhou & 257 & 21.84 & $19.95(17.77-22.32)$ & 189 & 10.99 & $10.67(9.28-12.24)$ \\
\hline Hainan & 217 & 21.38 & $18.36(16.14-20.81)$ & 179 & 11.65 & $11.41(9.89-13.12)$ \\
\hline Hebei & 510 & 31.25 & $31.85(29.53-34.26)$ & 425 & 18.81 & $19.37(17.72-21.12)$ \\
\hline Heilongjiang & 308 & 34.41 & $33.64(30.49-36.95)$ & 441 & 25.34 & $21.83(19.87-23.93)$ \\
\hline Inner Mongolia & 243 & 26.91 & $21.77(19.23-24.54)$ & 240 & 16.91 & $16.78(14.82-18.94)$ \\
\hline Qinghai & 418 & 34.60 & $30.50(27.84-33.29)$ & 281 & 19.71 & $20.52(18.37-22.85)$ \\
\hline Xinjiang & 241 & 19.13 & $17.03(15.06-19.19)$ & 179 & 9.82 & $9.84(8.52-11.35)$ \\
\hline Yunnan & 252 & 47.91 & $46.20(41.85-50.60)$ & 397 & 29.92 & $28.10(25.66-30.68)$ \\
\hline$p$ value & & $<0.001$ & $<0.001$ & & $<0.001$ & $<0.001$ \\
\hline
\end{tabular}

* The adjusted prevalence of HUA was adjusted for age and residential areas. a: low (1-40 g/day), moderate (41-60 g/day), and high (>60 g/day) for males; 1-20 g/day, 21-40 g/day, and >40 g/day for females. HUA, hyperuricemia; CNHS, China National Health Survey; MRF, modifiable risk factor; $\mathrm{Cl}$, confidence interval.

Overweight/Obesity Population

Attributable Fraction of Hyperuricemia
Obes Facts $2022 \cdot 15 \cdot 216-227$ DOI: $10.1159 / 000521163$ 


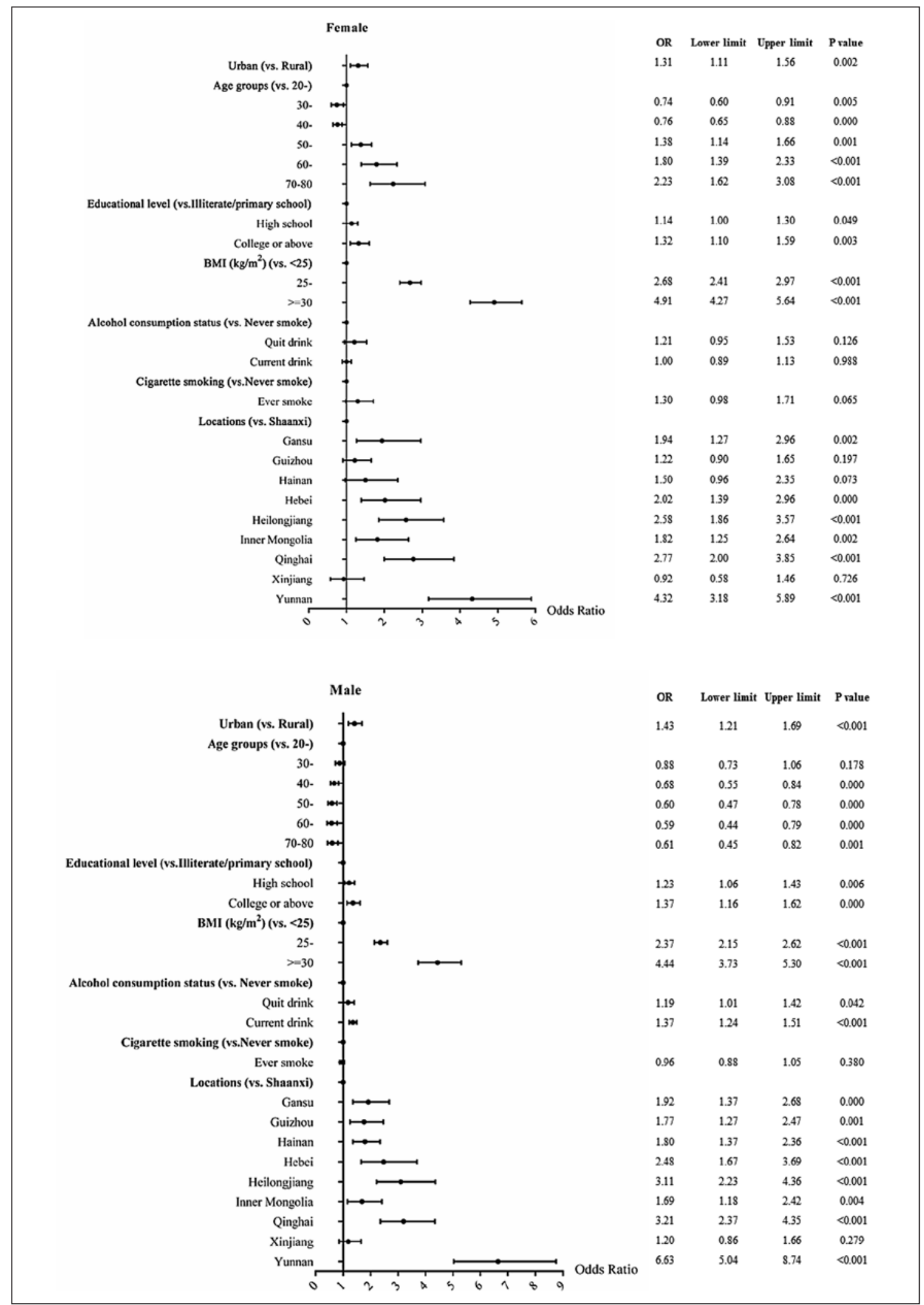

Fig. 1. The forest plot yielded by the logistic regression models to reflect the associated factors of hyperuricemia in different sexes. BMI, body mass index; OR, odds ratio. 


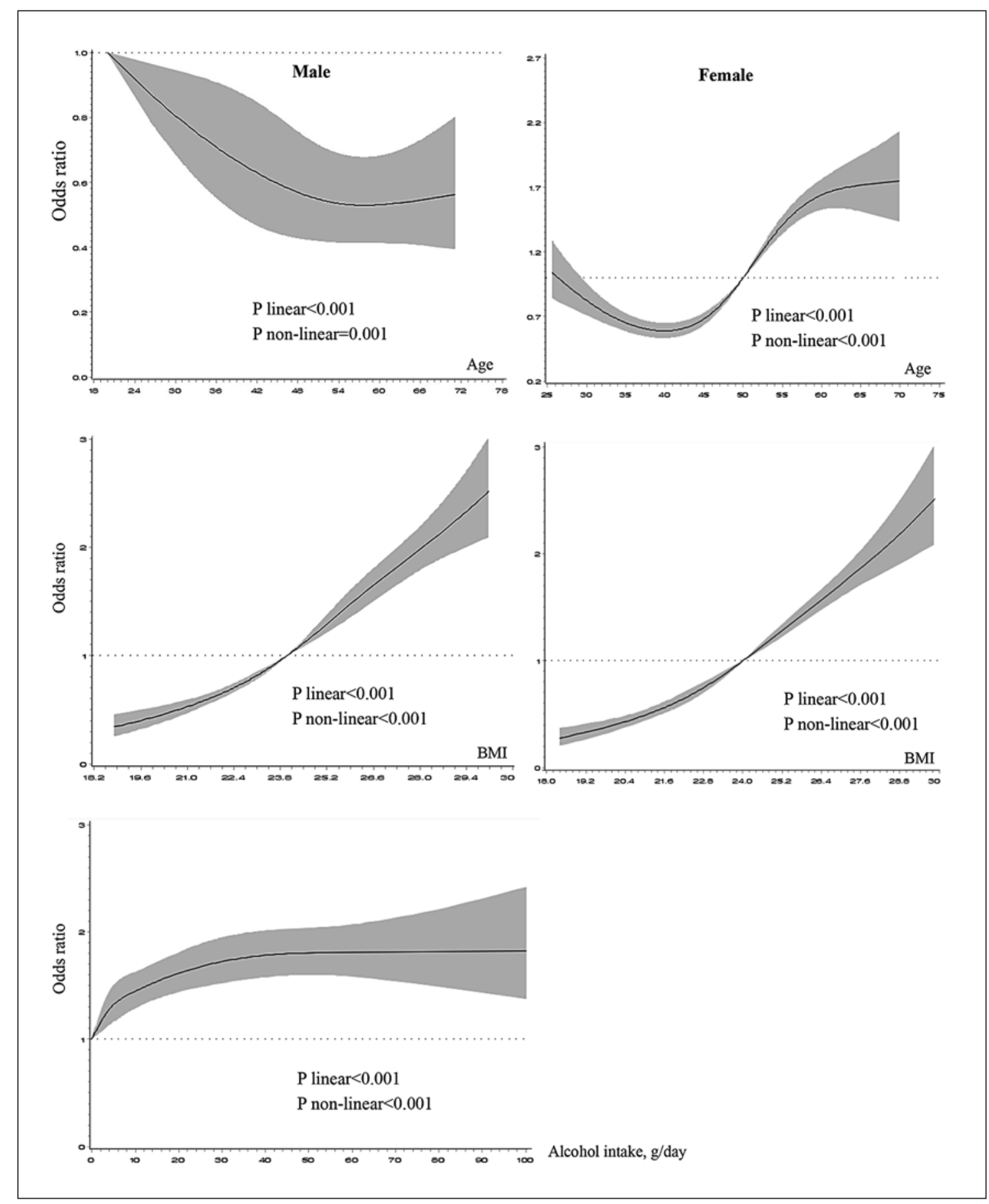

Fig. 2. RCS models reflecting the association between age, MRFs, and the OR of HUA stratified by sex. MRFs in male were BMI and alcohol consumption, and in female was BMI. The solid lines represent the predicted value, and the shadows were their 95\% CI. The adjusted factors were residential areas, education attainment, and study sites. RCS, restricted cubic-spline; MRF, modifiable risk factor; OR, odds ratio; HUA, hyperuricemia; BMI, body mass index; CI: confidence interval. 


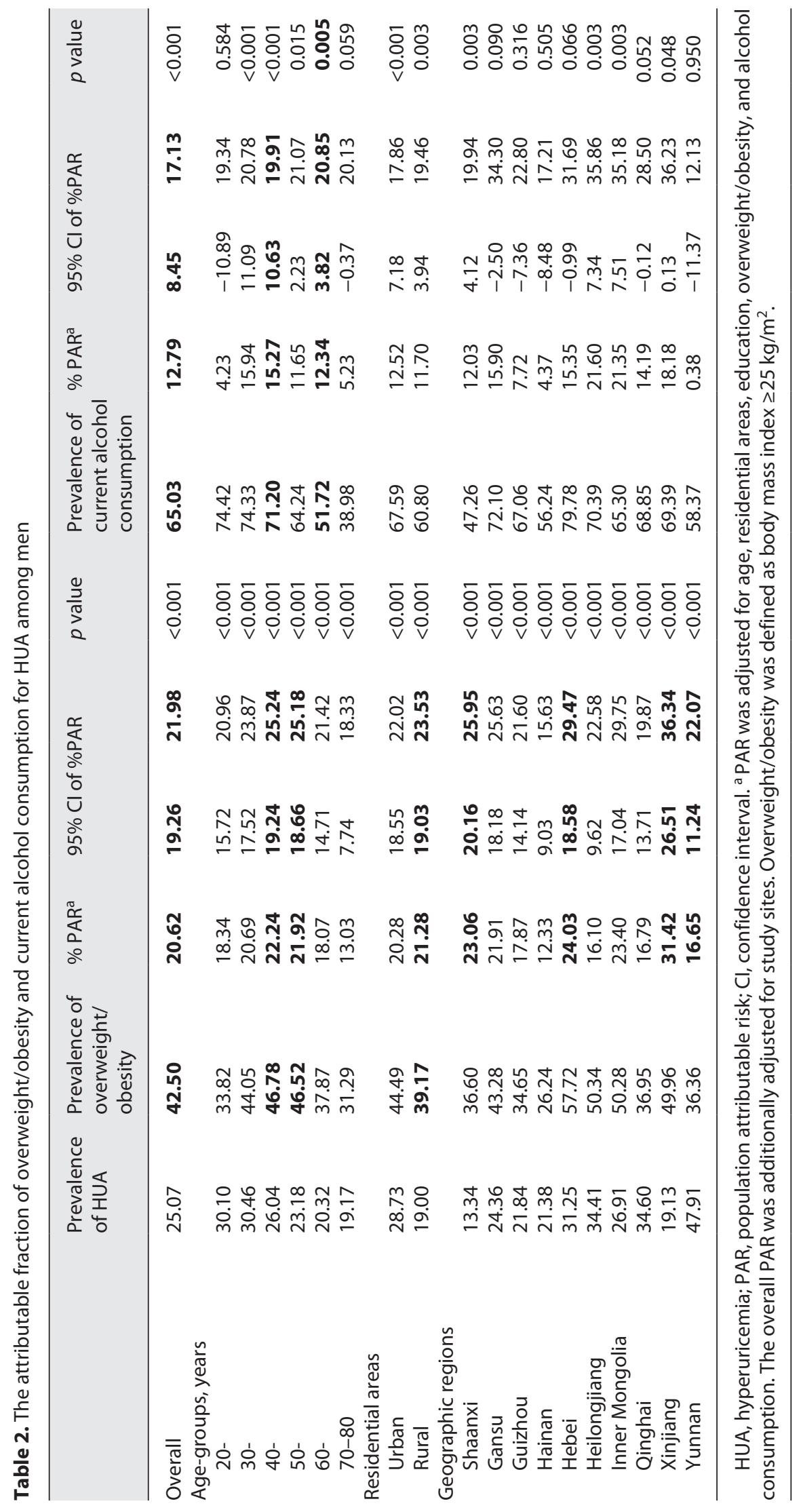


Table 3. The attributable fraction of overweight/obesity for HUA among women

\begin{tabular}{|c|c|c|c|c|c|c|}
\hline & Prevalence & Prevalence of & $\%$ PAR $^{a}$ & $95 \% \mathrm{Cl}$ & f $\%$ PAR & $p$ value \\
\hline Overall & 15.94 & 32.14 & 18.08 & 17.13 & 19.02 & $<0.001$ \\
\hline Age-groups, years & & & & & & \\
\hline $20-*$ & 10.84 & 18.25 & 12.73 & 11.88 & 13.59 & $<0.001$ \\
\hline $40-$ & 11.35 & 31.53 & 17.90 & 15.83 & 19.97 & $<0.001$ \\
\hline $50-$ & 19.65 & 39.76 & 18.20 & 15.71 & 20.69 & $<0.001$ \\
\hline $60-80 *$ & 24.12 & 42.05 & 22.67 & 20.44 & 24.89 & $<0.001$ \\
\hline Residential areas & & & & & & \\
\hline Urban & 17.55 & 30.40 & 16.83 & 15.76 & 17.90 & $<0.001$ \\
\hline Rural & 12.98 & 35.32 & 20.55 & 18.64 & 22.45 & $<0.001$ \\
\hline Geographic regions & & & & & & \\
\hline Shaanxi & 9.07 & 29.33 & 15.77 & 12.70 & 18.85 & $<0.001$ \\
\hline Gansu & 16.36 & 27.70 & 15.33 & 13.12 & 17.54 & $<0.001$ \\
\hline Guizhou & 10.99 & 29.38 & 19.39 & 16.61 & 22.18 & $<0.001$ \\
\hline Hainan & 11.65 & 17.47 & 10.18 & 8.05 & 12.30 & $<0.001$ \\
\hline Hebei & 18.81 & 45.25 & 27.44 & 23.92 & 30.96 & $<0.001$ \\
\hline Heilongjiang & 25.34 & 39.83 & 22.46 & 19.49 & 25.42 & $<0.001$ \\
\hline Inner Mongolia & 16.91 & 35.84 & 18.95 & 14.80 & 23.09 & $<0.001$ \\
\hline Qinghai & 19.71 & 25.36 & 13.00 & 10.32 & 15.68 & $<0.001$ \\
\hline Xinjiang & 9.82 & 38.62 & 25.88 & 21.84 & 29.93 & $<0.001$ \\
\hline Yunnan & 29.92 & 29.86 & 13.83 & 11.20 & 16.45 & $<0.001$ \\
\hline
\end{tabular}

HUA, hyperuricemia; PAR, population attributable risk; $\mathrm{Cl}$, confidence interval. * The number of HUA cases in the 20-29 and 70-80 years groups among female was small, so we combined them with the next age-group to get a more stable estimation. Overweight/

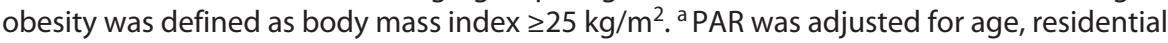
areas, and overweight/obesity. The overall PAR was additionally adjusted for study sites. people from west China, i.e., Xinjiang and Shaanxi, where the prevalence of MRFs were also high, had the lowest HUA prevalence in both sexes. Subjects from Yunnan had the highest adjusted HUA prevalence, with $46.2 \%$ (41.9-50.6\%) in men and $28.1 \%(25.7-30.7 \%)$ in women, i.e., nearly half of men and one-third of women from Yunnan province had HUA.

\section{The Population- and Geographic-Varied Importance of HUA MRFs}

PARs were estimated to reflect the importance of each risk factor in the subpopulation and areas (shown in Tables 2, 3; Fig. 3). The overall PAR of overweight/obesity in men was $20.6 \%(19.3 \%-22.0 \%)$. This number was similar in women, which was $18.1 \%(17.1 \%-19.0 \%)$. Around $12.8 \%(8.5 \%-17.1 \%)$ HUA cases in men were due to current alcohol consumption. PARs in urban and rural areas were also presented in Tables 2 and 3.

The prevalence of HUA in each area was predicted if all MRFs were removed (Fig. 3). There were substantial regional differences on PAR. For instants, almost half (44.8\%) of HUA male cases from North China (Inner
Mongolia) were attributable to overweight/obesity and alcohol consumption; in contrary, only $12.3 \%$ HUA male cases from South China (Hainan province) can be attribute to excess body weight. In middle China, Hebei province, where people had a relatively high prevalence of excess body weight $(57.7 \%)$ and alcohol consumption (79.8\%), HUA prevalence could be reduced by $24 \%$ if all MRFs were removed (decreased to the reference level, i.e., normal weight and no alcohol consumption) (Fig. 3).

\section{Discussion}

In this large population-based study, using data from the CNHS, we revealed the high prevalence of HUA (defined as SUA $>417 \mu \mathrm{mol} / \mathrm{L}$ for men and $>340 \mu \mathrm{mol} / \mathrm{L}$ for women) and its geographic variation. Specifically, the prevalence of HUA in men from Southwest China (Yunnan province) was $48 \%$. We also found the populational and regional differences in the importance of MRFs to HUA.

Although HUA is a common health condition, the epidemiology varied greatly among areas and populations 

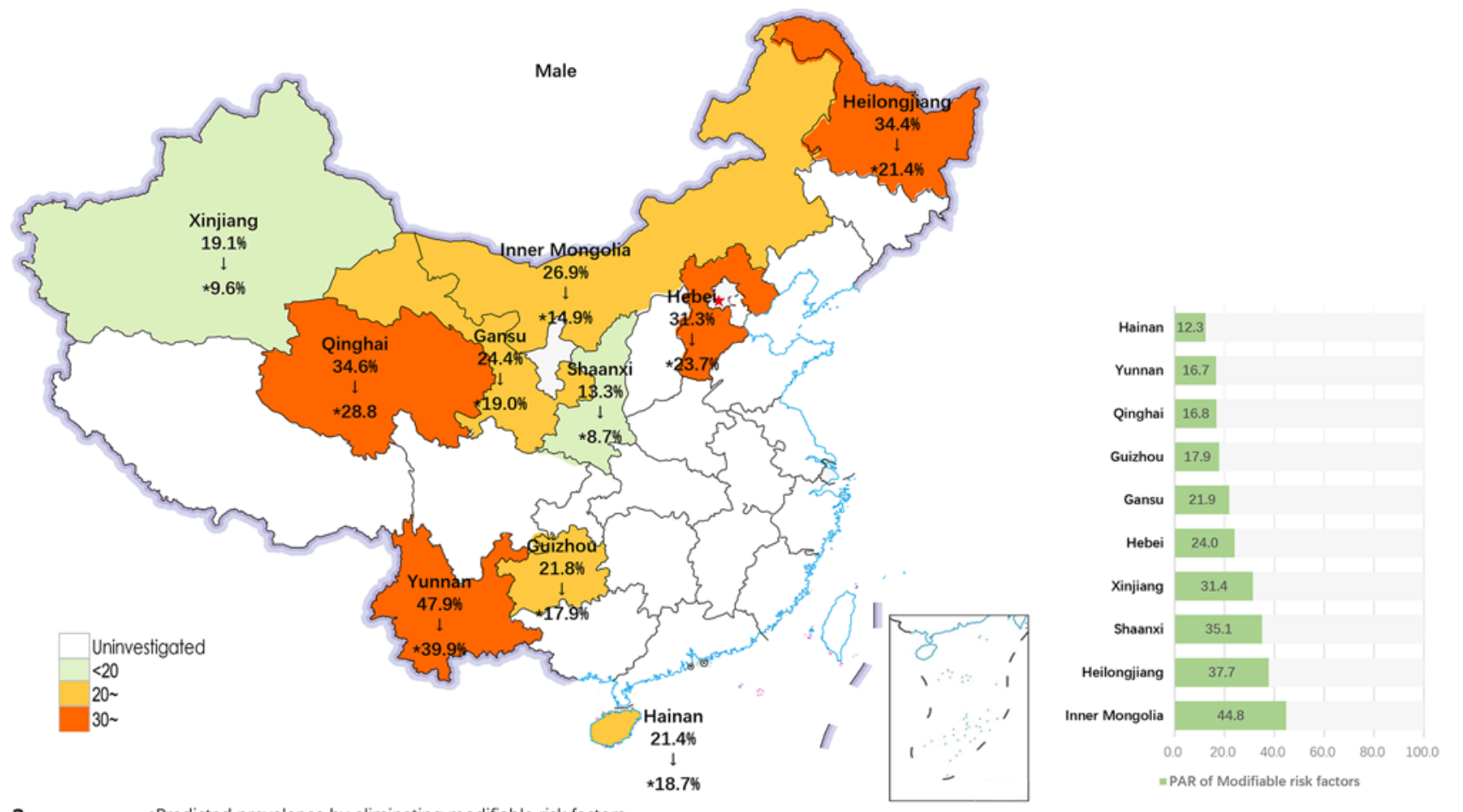

a

*Predicted prevalence by eliminating modifiable risk factors
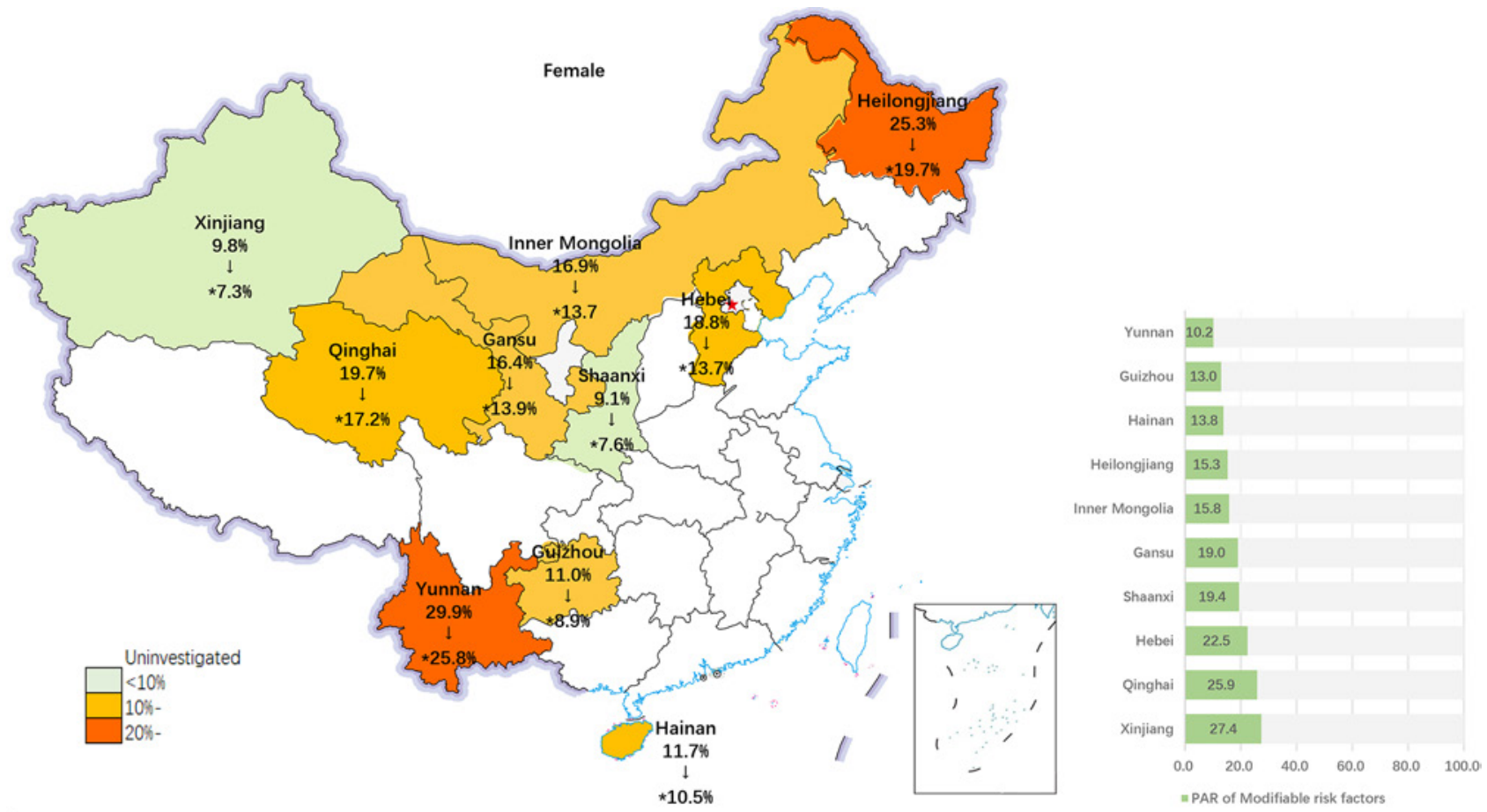

b

*Predicted prevalence by eliminating modifiable risk factors

Fig. 3. The geographic disparity on HUA prevalence and PAR (\%) of MRFs among adults from the CNHS, 20122017. a Male: the MRFs were overweight/obesity and alcohol consumption. b Female: the MRF was overweight/ obesity. PAR was adjusted for age, residential areas, and education attainment. The overall PAR was additionally adjusted for study sites. HUA, hyperuricemia; MRF, modifiable risk factor; PAR, population attributable risk; CNHS, China National Health Survey. 
$[6,28,29]$. Data from NHANES implied over 20\% HUA prevalence among US general population during 20152016 [28], and this number was $8.4 \%$ according to a national survey in Chinese adults in 2009-2010 [29]. A meta-analysis of pooled data from 2000 to 2014 presented a $13.3 \%$ prevalence of HUA among adults in mainland China, with a varied number in different areas [6]. Our data, especially in men, showed much higher HUA prevalence than other studies conducted in China [6-8]. This may attribute to the study population heterogeneity. As one of the main purposes of the CNHS, the populations from different geographic areas with a multicultural background and lifestyles were selected to understand the geographic and socioeconomic variations on health profiles. Study sites from Southwest China [30] may increase the overall prevalence of HUA, thus resulting in inconsistency among studies.

As BMI alone explains most of the variation of SUA levels and attributes most to the risk of HUA $[12,25]$, the prevalence of excess body weight may explain partially of the geographic variation of HUA burden. In the study population, especially in men, the prevalence of overweight/obesity was from $20.7 \%$ to $56.8 \%$. As the prevalence of HUA was much higher in males than in females, the problem of excess body weight in men was challenging for a SUA-lowering effort. The situation was not optimistic in females either. The data showed an overall $32.1 \%$ proportion of overweight/obesity in the female subjects, of which around $40 \%$ was in the North provinces Hebei and Heilongjiang. Generally, MRFs, such as excess body weight and alcohol consumption, are more prevalent in North or west China than in the South [3134]. Both excess body weight and alcohol use are risk factors for HUA, gout, and other cardiometabolic diseases [12, 35-37] and have a complicated interrelated impact [8]. Giving the high prevalence of overweight/obesity, alcohol use, and HUA, more attentions should be paid to improve the public health status.

Although the level of SUA is influenced by both genetic and environmental factors [38], intervention on MRFs has important public health significance, given the hypothesis that disease burden in a population may be reduced by eliminating one or more of these risk factors. The calculation of PAR of MRFs could reflect the level of exposure contribution by incorporating both its effect size and prevalence [25], thus could reflect the potential achieved health benefits when these risk factors were removed by initiating public health promotion strategies. Revealed by our study, the proportion of cases that could be avoided by removing MRFs varied geographically. Men in Inner Mongolia would have been avoid-

Overweight/Obesity Population

Attributable Fraction of Hyperuricemia ed $44.8 \%$ of HUA cases by weight loss (reduced BMI to at least $<25 \mathrm{~kg} / \mathrm{m}^{2}$ ) together with quit alcohol drinking. In contrast, men in Yunnan province, although they had the highest HUA prevalence, the potential effect of reducing HUA disease burden by weight loss or quit drinking would not be optimistic. The same situation also existed for women from the South China (Hainan province). For areas with high prevalence of HUA but low PAR of MRFs, other reasons that lead to the disease onset should be further studied, such as genetic susceptibility and other environmental factors.

Excess body weight increases HUA risk by raising the SUA level through decreased renal SUA excretion and increased SUA production $[39,40]$. Weight loss could reduce SUA [14]. The current study also demonstrated that BMI had a higher PAR than alcohol consumption, which was around $20 \%$ in both sexes. In other words, nearly $20 \%$ cases in the study population would have keep normal SUA levels if their BMI decreased to less than $25 \mathrm{~kg} / \mathrm{m}^{2}$. Drinking alcohol has a long history in Chinese culture, the China Kadoorie Biobank study revealed that $76 \%$ of men and $36 \%$ of women reported drinking alcohol within 1 year [41]. Although previous studies have shown that alcohol use was a risk factor for HUA and gout [13, 38], different regions may have varied effect size. In addition, the effect size is related to the intake dose and types of alcoholic drink, e.g., beer may have a stronger effect in increasing SUA than that of liquor [38]. In this study, the predominant alcoholic drink was liquor, taking account for over $90 \%$ of the daily alcohol intake in each study site. The "healthy worker effect" may also explain the low PAR of alcohol consumption. People suffering from chronic diseases may quit or reduce the amount of alcohol consumption, thus leading to conservative estimates.

Although guidelines for the treatment of HUA and gout in mainland China have been developed [42], the understanding on how this should be integrated and customized with local conditions is rare and lack of evidence. Findings of our study suggested that for areas with higher PAR of MRFs, the weight loss and alcohol reduction intervention should be strengthened to decrease the HUA prevalence; but, for areas with lower PAR, paralleled with lifestyle intervention, etiology research should be further initiated to understand the predominant reason for disease onset.

Strengths and limitations of our study should be acknowledged. Using a large representative sample from different areas, the results can be more reliable to demonstrate how the population impact of MRFs varied geographically. Another strength is that throughout the survey, we used the same equipment and stringent and consistent criteria for measurement, thus making the results from different study sites com- 
parable. Third, by calculating the adjusted PAR, we estimated the fraction of cases that would be avoided by eliminating the MRFs from the population and assessed the relative importance of these factors in different areas, which could provide key information for precise disease prevention.

Unlike prospective studies, limited by the cross-sectional design, the result may lead to uncertainty regarding the temporal sequence of exposure-outcome associations. If some participants modified their lifestyle because of health conditions, the effect size between risk factors and the outcome would be underestimated, leading to conservative results. Moreover, information on some environmental factors related with HUA was not collected, such as dietary [37] and diuretic medicine intake. However, as previous studies suggested, there was an indirect effect of diet and exercise on the risk of HUA, which means when BMI was adjusted in the association model, their association disappeared $[25,43]$; meanwhile the direct effect of diet is likely smaller than the indirect effect through BMI [44].

\section{Conclusion}

This study indicates that there were sex and geographic variations on the prevalence and role of MRFs (excess body weight and alcohol consumption) of HUA. These findings call for more tailored and local customized prevention strategies. In areas with low PAR of MRFs but high prevalence of HUA, further research is necessary for understanding the etiology of HUA.

\section{Acknowledgments}

We appreciate all the participants of CNHS and gratefully thank all staff members from the ten provinces who contributed considerable time and energy for this survey. We also thank Dr. Wei Han for his help with statistical analyses and thank Xiaoyang Wang, Jia Zhang, and Huiru Ren for their effects in the field work.

\section{Statement of Ethics}

The study has been carried out in accordance with the Declaration of Helsinki. Ethical approval was obtained from the Bioethical Committee of Institute of Basic Medical Sciences, Chinese Academy of Medical Sciences (No. 029-2013). All participants provided written informed consent before the survey.

\section{Conflict of Interest Statement}

The authors have no conflicts of interest to declare.

\section{Funding Sources}

This work was supported by the National Key R\&D Program of China (grant no. 2016YFC0900600/2016YFC0900601), the National Natural Science Foundation of China (Grant No. 82003531), and the Key Basic Research Program of the Ministry of Science and Technology of China (Grant No. 2013FY114100).

\section{Author Contributions}

H.H. and G.S. contributed to conceptualization; H.H. contributed to methodology, software, formal analysis, and writing - original draft preparation; H.H., G.S., and L. Pan contributed to validation; L. Pan, X.R., D.W., J.D., Z.C., H.W., X.W., F.L., L. Pa, X.P., Y.W., C.Y., G.S., and H.H. contributed to investigation; G.S., H.H., X.R., D.W., J.D., Z.C., H.W., X.W., F.L., L. Pa, X.P., Y.W., and C.Y. contributed to resources; H.H. and G.S. contributed to data curation; G.S. contributed to writing - review and editing; H.H., Y.W., and C.Y. contributed to visualization; G.S. and L. Pan contributed to supervision; G.S., L. Pan, and H.H. contributed to project administration; G.S. and H.H. contributed to funding acquisition. All the authors read and approved the final manuscript.

\section{Data Availability Statement}

The datasets generated or analyzed for this study are available from the corresponding author by reasonable request.

\section{References}

1 Feig DI, Kang DH, Johnson RJ. Uric acid and cardiovascular risk. N Engl J Med. 2008;359: 1811-21.

2 Kuo CF, Grainge MJ, Zhang W, Doherty M. Global epidemiology of gout: prevalence, incidence and risk factors. Nat Rev Rheumatol. 2015;11:649-62.
3 Kim Y, Kang J, Kim GT. Prevalence of hyperuricemia and its associated factors in the general Korean population: an analysis of a population-based nationally representative sample. Clin Rheumatol. 2018;37:2529-38.

4 Trifiro G, Morabito P, Cavagna L, Ferrajolo C, Pecchioli S, Simonetti M, et al. Epidemiology of gout and hyperuricaemia in Italy during the years 2005-2009: a nationwide populationbased study. Ann Rheum Dis. 2013;72:694-700.
5 Zhu Y, Pandya BJ, Choi HK. Prevalence of gout and hyperuricemia in the US general population: the National Health and Nutrition Examination Survey 2007-2008. Arthritis Rheum. 2011;63:3136-41.

6 Liu R, Han C, Wu D, Xia X, Gu J, Guan H, et al. Prevalence of hyperuricemia and gout in Mainland China from 2000 to 2014: a systematic review and meta-analysis. Biomed Res Int. 2015;2015:762820. 
7 Song P, Wang H, Xia W, Chang X, Wang M, An L. Prevalence and correlates of hyperuricemia in the middle-aged and older adults in China. Sci Rep. 2018;8:4314.

8 Wu J, Qiu L, Cheng XQ, Xu T, Wu W, Zeng $\mathrm{XJ}$, et al. Hyperuricemia and clustering of cardiovascular risk factors in the Chinese adult population. Sci Rep. 2017;7:5456.

9 Miao Z, Li C, Chen Y, Zhao S, Wang Y, Wang Z, et al. Dietary and lifestyle changes associated with high prevalence of hyperuricemia and gout in the Shandong coastal cities of Eastern China. J Rheumatol. 2008;35: 1859.

10 Wang R, Tang Z, Sun F, Diao LJ. [Prevalence of hyperuricemia in the elderly in 7 areas of China]. Zhonghua Liu Xing Bing Xue Za Zhi. 2018;39:286-8. Chinese.

11 Lyngdoh T, Vuistiner P, Marques-Vidal P, Rousson V, Waeber G, Vollenweider P, et al. Serum uric acid and adiposity: deciphering causality using a bidirectional Mendelian randomization approach. PLoS One. 2012;7: e39321.

12 Oikonen M, Wendelin-Saarenhovi M, Lyytikäinen LP, Siitonen N, Loo BM, Jula A, et al. Associations between serum uric acid and markers of subclinical atherosclerosis in young adults. The cardiovascular risk in Young Finns study. Atherosclerosis. 2012; 223:497-503.

13 Jee YH, Jung KJ, Park YB, Spiller W, Jee SH. Causal effect of alcohol consumption on hyperuricemia using a Mendelian randomization design. Int J Rheum Dis. 2019;22:1912-9.

14 Nielsen SM, Bartels EM, Henriksen M, Wæhrens EE, Gudbergsen H, Bliddal H, et al. Weight loss for overweight and obese individuals with gout: a systematic review of longitudinal studies. Ann Rheum Dis. 2017;76:187082.

15 Rockhill B, Newman B, Weinberg C. Use and misuse of population attributable fractions. Am J Public Health. 1998;88:15-9.

16 He H, Pan L, Pa L, Cui Z, Ren X, Wang D, et al. Data resource profile: the China National Health Survey (CNHS). Int J Epidemiol. 2018; 47:1734-5f.

17 Centers for Disease Control and Prevention. National Health and Nutrition Examination Survey: reference manuals and report [Internet]. Atlanta: Centers for Disease Control and Prevention. [cited 2020 Jan 31]. Available from: https://wwwn.cdc.gov/nchs/nhanes/ nhanes3/ManualsAndReports.aspx.

18 World Health Organization. Obesity and overweight [Internet]. Geneva: World Health Organization. [cited 2019 Apr 12]. Available from: www.who.int/news-room/fact-sheets/ detail/obesity-and-overweight.

19 He H, Pan L, Cui Z, Sun J, Yu C, Cao Y, et al. Smoking prevalence, patterns, and cessation among adults in Hebei Province, Central China: implications From China National Health Survey (CNHS). Front Public Health. 2020;8:177.

20 World Health Organization. International guide for monitoring alcohol consumption and related harm [Internet]. Geneva: World Health Organization. [cited 2019 May 18]. Available from: https://apps.who.int/iris/bitstream/handle/10665/66529/WHO_MSD_ MSB_00.4.pdf;sequence=1.

21 Ma YC, Zuo L, Chen JH, Luo Q, Yu XQ, Li Y, et al. Modified glomerular filtration rate estimating equation for Chinese patients with chronic kidney disease. J Am Soc Nephrol. 2006; 17:2937-44.

22 Goicoechea M, de Vinuesa SG, Verdalles U, Ruiz-Caro C, Ampuero J, Rincón A, et al. Effect of allopurinol in chronic kidney disease progression and cardiovascular risk. Clin J Am Soc Nephrol. 2010;5:1388-93.

23 Kang E, Hwang SS, Kim DK, Oh KH, Joo KW, Kim YS, et al. Sex-specific relationship of serum uric acid with all-cause mortality in adults with normal kidney function an observational study. J Rheumatol. 2017;44:380-7.

24 Roalfe AK, Holder RL, Wilson S. Standardisation of rates using logistic regressions: a comparison with the direct method. BMC Health Serv Res. 2008;8:275.

25 Choi HK, McCormick N, Lu N, Rai SK, Yokose C, Zhang Y. Population impact attributable to modifiable risk factors for hyperuricemia. Arthritis Rheumatol. 2020;72:157-65.

26 Zou G. A modified poisson regression approach to prospective studies with binary data. Am J Epidemiol. 2004;159:702-6.

27 SAS Support. Estimating attributable risks when covariates are present using logistic and other models [Internet]. [cited 2021 May 18]. Available from: https://support.sas.com/ $\mathrm{kb} / 63 / 471 . \mathrm{html}$.

28 Chen-Xu M, Yokose C, Rai SK, Pillinger MH, Choi HK. Contemporary prevalence of gout and hyperuricemia in the United States and decadal trends: the National Health and Nutrition Examination Survey, 2007-2016. Arthritis Rheumatol. 2019;71:991-9.

29 Liu H, Zhang XM, Wang YL, Liu BC. Prevalence of hyperuricemia among Chinese adults: a national cross-sectional survey using multistage, stratified sampling. J Nephrol. 2014;27:653-8.

30 Zhang X, Meng Q, Feng J, Liao H, Shi R, Shi $\mathrm{D}$, et al. The prevalence of hyperuricemia and its correlates in Ganzi Tibetan Autonomous Prefecture, Sichuan Province, China. Lipids Health Dis. 2018;17:235.

31 Tong X, Wang X, Wang D, Chen D, Qi D, Zhang $\mathrm{H}$, et al. Prevalence and ethnic pattern of overweight and obesity among middleaged and elderly adults in China. Eur J Prev Cardiol. 2019;26:1785-9.
32 Zhang L, Wang Z, Wang X, Chen Z, Shao L, Tian Y, et al. Prevalence of overweight and obesity in China: results from a cross-sectional study of 441 thousand adults, 2012-2015. Obes Res Clin Pract. 2020;14:119-26.

33 Zhang L, Wang Z, Wang X, Chen Z, Shao L, Tian Y, et al. Prevalence of abdominal obesity in China: results from a Cross-Sectional Study of nearly half a million participants. Obesity. 2019;27:1898-905.

34 Lyu J, Guo Y, Bian Z, Yu C, Wang Z, Zhou H, et al. Regional differences in patterns of alcohol consumption : findings from the China Kadoorie Biobank study on half a million people from 10 regions. Chin J Epidemiol. 2014;35:875-81.

35 Elagizi A, Kachur S, Lavie CJ, Carbone S, Pandey A, Ortega FB, et al. An overview and update on obesity and the obesity paradox in cardiovascular diseases. Prog Cardiovasc Dis. 2018;61:142-50.

36 Day E, Rudd JHF. Alcohol use disorders and the heart. Addiction. 2019;114:1670-8.

37 Rivera-Paredez B, Macías-Kauffer L, Fernandez-Lopez JC, Villalobos-Comparán M, Martinez-Aguilar MM, de la Cruz-Montoya A, et al. Influence of genetic and non-genetic risk factors for serum uric acid levels and hyperuricemia in Mexicans. Nutrients. 2019;11: 1336.

38 Ragab G, Elshahaly M, Bardin T. Gout: an old disease in new perspective-a review. J Adv Res. 2017;8:495-511.

39 Facchini F, Chen YD, Hollenbeck CB, Reaven $\mathrm{GM}$. Relationship between resistance to insulin-mediated glucose uptake, urinary uric acid clearance, and plasma uric acid concentration. JAMA. 1991;266:3008-11.

40 Fam AG. Gout, diet, and the insulin resistance syndrome. J Rheumatol. 2002;29:1350.

41 Millwood IY, Li L, Smith M, Guo Y, Yang L, Bian Z, et al. Alcohol consumption in 0.5 million people from 10 diverse regions of China prevalence, patterns and socio-demographic and health-related correlates. Int J Epidemiol. 2013;42:816-27.

42 Association CM. Guideline for primary care of gout and hyperuricemia: practice version (2019). Chin J Gen Pract. 2019;19:486-94. Chinese.

43 Williams PT. Effects of diet, physical activity and performance, and body weight on incident gout in ostensibly healthy, vigorously active men. Am J Clin Nutr. 2008;87:1480-7.

44 Dessein PH, Shipton EA, Stanwix AE, Joffe BI, Ramokgadi J. Beneficial effects of weight loss associated with moderate calorie/carbohydrate restriction, and increased proportional intake of protein and unsaturated fat on serum urate and lipoprotein levels in gout: a pilot study. Ann Rheum Dis. 2000;59:53943.
Overweight/Obesity Population

Attributable Fraction of Hyperuricemia
Obes Facts 2022;15:216-227 